the pace. But it will not indefinitely be possible to meet an unrestrained demand. Sooner or later it will be necessary for those who use computers to work out more explicit arrangements for making the most economical use of them. One difficulty is that the diversity of the demand on computer power will make it harder to operate the kind of committee system which has proved successful in managing the big accelerating machines. In any case, it is hard to see how a committee would ever be able to decide confidently between the merits of some complicated analysis in demography and a Monte Carlo calculation in nuclear physics.

The question remains of how the Computer Board may serve as a model for the development of sectors of research in British universities. The first need, of course, is that the board should show that it can function effectively. The special characteristics of computers, and the obvious need of integration within the system, also restrict the usefulness of comparison with other fields of research in which development is necessary. It is nevertheless tempting to wonder whether it will not turn out that the need for development in, say, molecular biology can best be met by an independent board given the means and the responsibility for seeing that developments at universities and other research establishments are sensibly co-ordinated -and blessed with funds. Evidently the Computer Board is more important than the narrowness of its field of interest would suggest.

\section{WE WUZ ROBBED}

The World Cup which has recently been enacted in Britain may have been fun to watch, but there is no question that it was a thoroughly badly designed experiment. If the intention was to find out which football team is the best in the world, the organizers would have gone about their task quite differently.

Just as the frequency of deaths from kicks by horses in those far-off but now classical stables of the Prussian Army are described by a Poisson distribution, so are the numbers of goals scored by the competing teams in the recent series of World Cup matches. Table 1 shows the numbers of occasions during the series of thirty-two games (each involving two teams) on which specified numbers of goals were scored, together with the frequencies described by a Poisson distribution of the form $P(n)=\mathrm{e}^{-q} q^{n} / n$ ! with $q=1 \cdot 234$, or the mean of the observed scores.

Numbers
of goals
0
1
2
3
4
5
More than 5

$\begin{array}{cc}\text { Table 1 } & \\ \text { Observed } & \text { Calculated } \\ 18 & 65 . P(n) \\ 20 & 18.4 \\ 15 & 22 \cdot 8 \\ 7 & 14 \cdot 0 \\ 2 & 6 \cdot 0 \\ 2 & 1 \cdot 8 \\ 0 & 0.5 \\ & 0.7\end{array}$

In reality an even better agreement between fact and prediction can be obtained by taking $q=1 \cdot 27$, a value which lies well within the standard deviation of the mean.

The mere fact that a Poisson distribution can describe so well the distribution of scores by individual teams goes a long way to suggest that the teams were much of a muchness in talent and their scores were independent of each other. From this point of view, the decision that the outcome of the whole competition should depend on the outcome of a single game between the two so-called finalists was as much of a farce as a great many West German supporters already know it to have been. If it is assumed that the goal scoring potentiality of the two teams is equally well described by the Poisson distribution already specified, the chance that the result will be a draw is a mere $0 \cdot 27$. In other words, if two teams are equally matched, the chance that the result will be an active injustice to one of them will be $0 \cdot 73$. By the same token, a team which is slightly less skilled than its opponent can nevertheless expect a one in three chance of winning the deciding match.

Chancy outcomes of individual games are given extraordinary importance by the overall design of the World Cup competition, in which small groups of teams play an incestuous competition on their own before one team is sent on to another stage in the competition. Obviously a properly randomized block design would be an improvement. But even within the framework of the existing competition, much could be done to reduce the gross sampling error under the present arrangements. Replication of the crucial games by a series of identical trials is one obvious remedy, and the organizers of the World Series of baseball games in the United States have in this spirit arranged that the winner should be the one who wins the most of seven games. The ideal is that in future World Cup competitions, the organizers should fix a certain limit of confidence (say, $P<0.01$ ) and then that they should require that the finalists go on playing against each other either until the superiority of one or the other of them is properly established, or until both parties agree to negotiate a draw.

A more practicable alternative might be to redesign the parameters of the game of football in such a way that a respectable degree of confidence in the outcome of the competition can be acquired in a reasonable interval of time. If, for example, it were agreed that single cup finals should remain, but that no team should be declared the winner until its score excecds that of its opponent by three standard deviations of Poisson distribution, it might be necessary to design the game of football so that it would be practicable for one side to score 100 goals or so within the limits of endurance of the spectators. This implies that the parameter $q$ would have to be much greater than under the present rules. Such a change could easily be brought about, possibly by widening the goalposts or by abolishing goalkeepers. The precise method by which these matters are attended to is, of course, much less important than that they should be dealt with quickly. The whole of South America knows that. 